\title{
Flight of the bumblebee
}

During flight, insects beat their wings hundreds of times per second, faster than would be possible if their flight muscles worked by contraction, like vertebrate muscles do. How they do this is a matter of some debate, but new data from an $\mathrm{x}$-ray scattering study suggest that insect flight muscle activation relies on a mechanism that is shared with vertebrate muscle.

In vertebrates, muscle contraction is based on calcium: calcium ions are released in response to a nerve signal and then captured by troponins attached to actin filaments, causing the filaments to rotate and expose myosin binding sites. Myosin binds to actin, then gets bent, pulling the actin filament and causing muscle to contract. This process burns energy-far too much to support the rapid beating of an insect's wings-and requires calcium pumping, which cannot occur fast enough to sustain flight. Instead of contracting, then, insect muscles respond to nerve signals by entering a state of selfsustained oscillation induced by 'stretch activation, a mechanism in which the force generated by the flight muscles increases as the muscles are extended. It isn't clear, however, what triggers stretch activation in insect flight muscles. Early work suggested that extension of the muscles exposed more myosin binding sites, but a more recent study proposed that insect flight muscles expressed a unique, calcium-independent form of troponin that enabled the muscle oscillations. Hiroyuki Imamoto, a biophysicist at Japan Synchrotron Radiation Research Institute (Hyogo), told Nature News, "It has long been known that many insects don't move their flight muscles in the way vertebrates do. The big question is whether the difference is unique to insect flight muscles or exploits a property common to all muscle proteins."

To address this question, Imamoto and his colleague Naoto Yagi placed female bumblebees (Bombus ignitus) in the path of an $\mathrm{x}$-ray beam and analyzed the beam scatter produced by their muscles during flight along with synchronized video footage, captured at 40 frames per wing beat, or 5,000 frames per second.

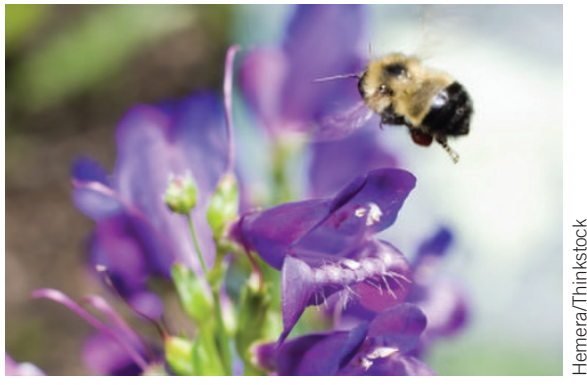

Imamoto and Yagi found that myosin twisted as the bees' flight muscles extended, enabling stronger binding to actin (Science published online 22 August 2013; doi:10.1126/science.1237266). This observation indicates that stretch activation is based on the same fundamental myosinactin interaction that underlies vertebrate muscle contraction. Instead of developing a new mechanism for the rapid muscle activation needed to fly, insects refined an existing mechanism for muscle force generation.

Monica Harrington

\section{ALZHEIMER'S DISEASE LINKED TO BRAIN COPPER BUILDUP}

Metals such as iron, copper and zinc are elevated in the brains of humans with Alzheimer's disease (AD) and those of some animal models of AD. Two recent studies now show that copper accelerates the pathology of AD by promoting the accumulation of the peptide amyloid- $\beta$ in the brain.

In the first study, Lisa M. Miller and researchers at Stony Brook University, NY, used synchrotron x-ray fluorescence microscopy to image metal ions in the brains of two AD mouse models. They compared the amyloid- $\beta$ plaques in $A D$ mice that exhibited neurodegeneration with those in $A D$ mice that showed little neurodegeneration. The plaques in mice with neurodegeneration contained $\sim 25 \%$ more copper (Biomedical Spectroscopy and Imaging 2, 129-139; 2013). These data suggest that impaired neuron function may result from an excess of copper in the brains of these mice, possibly indicating that cellular control of copper levels is altered in AD. Miller's group previously found high levels of copper in human AD plaques as well.

In the second study, a group of scientists led by Rashid Deane (University of Rochester Medical Center, NY) gave very small amounts of copper, equivalent to what people would consume in a normal diet, to healthy mice over a 3-month period. The copper made its way into the blood and accumulated in the vessels that carry blood to the brain, especially in the cellular walls of the capillaries (Proc. Natl. Acad. Sci. USA published online 19 August 2013; doi:10.1073/pnas.1302212110). These capillary cells play an important role in preventing the copper from entering the brain. Lipoprotein receptor-related protein 1 (LRP1), which lines the capillaries, binds with amyloid- $\beta$, escorting it into the blood vessels and removing it from the brain. Over time, however, the accumulated copper disrupted the function of LRP1 through oxidation. Furthermore, copper in the brains of the mice stimulated neurons to increase their production of amyloid- $\beta$ and caused it to bind together in larger complexes. Finally, it provoked inflammation of the brain tissue, promoting the breakdown of the blood-brain barrier.

Copper is ubiquitous in the food supply and also can be found in drinking water and nutritional supplements. Although it appears to be one of the main environmental factors implicated in AD, scientists do not suggest copper be avoided altogether, as certain amounts can be beneficial to health. "The key will be striking the right balance between too little and too much copper consumption," says Deane. 